\title{
Cooling of Active Components in Structurally Integrated Phased Arrays Antennas
}

\author{
- EMuS 2019 - \\ Jens Leiß*, Thomas Ebert ${ }^{\dagger}$, Marta Martínez-Vázquez ${ }^{\dagger \dagger}$ and Rens Baggen ${ }^{\dagger \dagger}$ \\ ${ }^{*},+$ IMST GmbH \\ Carl-Friedrich-Gauß-Str. 2-4, 47475 Kamp-Lintfort \\ e-mail: leiss@imst.de,web page: http://www.imst.com \\ †IQ evolution $\mathrm{GmbH}$ \\ Steinbachstraße 15, 52074 Aachen \\ Email: t.ebert@iq-evolution.com, web page: http://www. iq-evolution.com
}

Key words: 3D printing, embedded cooling, additive manufacturing, 5G, Satcom

Abstract. This paper presents an innovative cooling solution for active phased array antennas, using a 3D printed liquid cooling device integrated in the structure's PCB.

\section{INTRODUCTION}

The need for higher integration of active components on multilayer printed circuit boards (PCB) has dramatically increased over the last decades. In particular, the demand for steerability of the antennas used for communication systems and the increase in their opering frequencies has brought phased arrays antennas into the forefront of antenna development. Unfortunately the efficiency of these components for higher frequencies is very low, so that only 5 to $10 \%$ of the consumed power is used for the radiated signal. The rest is dissipated as thermal losses. The result is a higher integration density of active components with low efficiency, which in turn means a higher density of thermal discharge in a very small area.

This heat produced by the active components needs to be dissipated from the circuits, to avoid damages. The common way to do this is by integrating a heat-spreader, mounting passive or active coolers onto the devices or to integrate a copper core in the PCB (Printed Circuit Board). These solutions limit the space available for routing, or are bulky and heavy. In this work an alternative solution for the thermal management of an active array in the Xband $(8-12 \mathrm{GHz})$ antenna PCB is introduced. This array is to be integrated in the structure of an aircraft, in the frame of the Horizon 2020 project ACASIAS [1]. The idea is to integrate a 3D-printed active cooling structure directly into the PCB. This printed structure can be custom tailored to the needs of the active Radio Frequency (RF) circuits.

A nickel-based cooler is manufactured using a Selective Laser Melting (SLM) process and integrated in a standard RF- multilayer PCB. A cooling liquid (e.g. deionised water) can then be pumped in the cooler to transfers the heat from the critical points of RF-circuits to a radiator outside of the antenna. This solution allows really high power dissipation (up to 1 
$\mathrm{kW}$ ). This paper also presents the measurements of the first prototype of a PCB with embedded the liquid-cooling.

\section{3D - METAL PRINTING - THE SLM-PROCESS}

In the Selective Laser Melting (SLM) process, summarised in Figure 1, 3D-metallic components are produced from a metal powder bed. A metal powder, in this case nickel (Ni), is applied layer by layer in an isolated chamber. Each powder layer is around $30 \mu \mathrm{m}$ in thickness. A laser beam moves over the powder layers, causing the material to melt locally to create solid metal corresponding to shapes of the 3D CAD model of the component. After one laser sweep, the manufacturing platform is lowered and a new powder layer is applied. Step by step, layer by layer, a homogeneous metal structure is build. With this process it is possible to build high resolution, completely closed structures, which would be impossible using other manufacturing processes [2], [3].

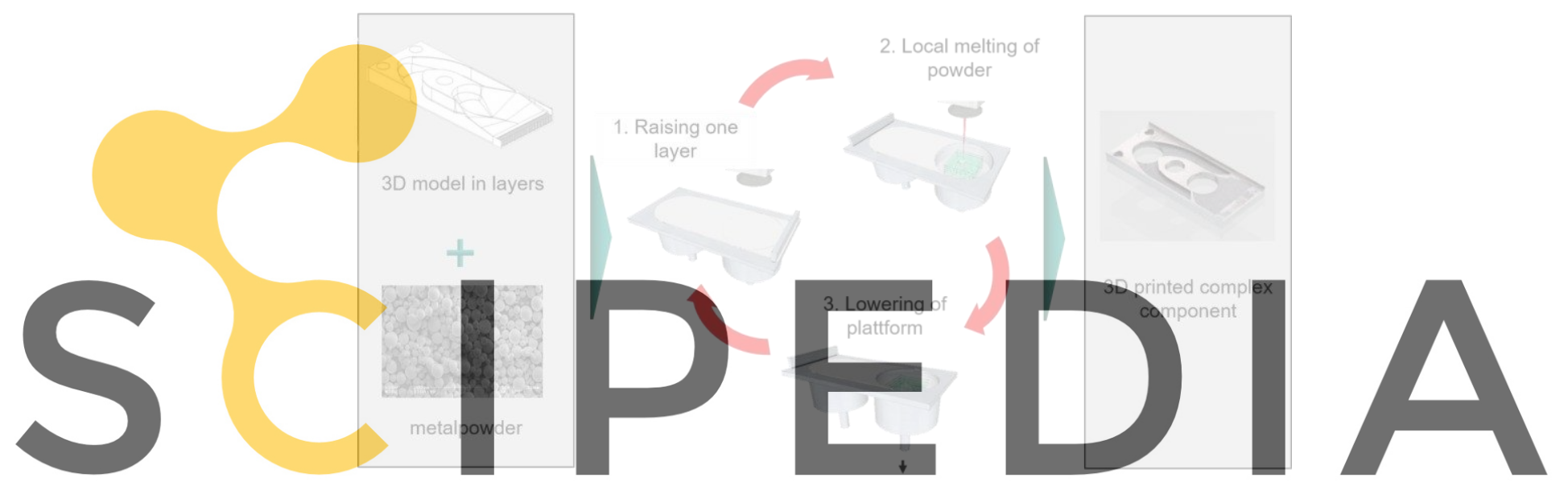

Figure 1: SLM-process - Manufacturing principle.

Register for free at https//www.scipedia.com to download the version without the watermark

\section{PCB WITH EMIBEDED Ni-COOLER}

A Ni-cooler with high thermal dissipation capability was manufactured (Figure 2) using the SLM process. This cooler can be embedded in FR4 material and integrated into a multilayer PCB structure, as needed for the implementation of an active phased array. The device must be robust enough to survive standard processes of the PCB industry, in particular, the different pressing cycles. Also, it has to allow for electrical connections between the PCB layers and be thin enough to be laminated in the PCB and to keep the inter-layer RF connections as short as possible.
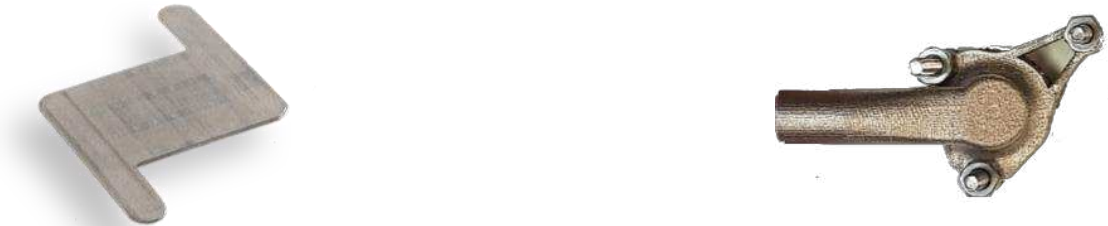

Figure 2: Left: $800 \mu \mathrm{m}$-thick 3D-SLM-printed Ni-cooler; Right: 3D-SLM-liquidconnector 
The advantage of these 3D Ni-coolers is that they can be customised taking into account the placement of the active components on the PCB. It is possible then to place the cooling channels directly under the components that are generating the heat, while still leaving enough space for routing and interconnections. Figure 3 shows the schematic buildup of a PCB with the integrated liquid cooling system. In- and outlet connections used to pump the cooling liquid (deionized water) into the system are placed in the top side of the PCB. The Nicooler is placed under the active, heating components, in this case RF power amplifiers (PA). The top and bottom layers of the PCB are connected with different types of metallized via holes, for signal and ground connections.

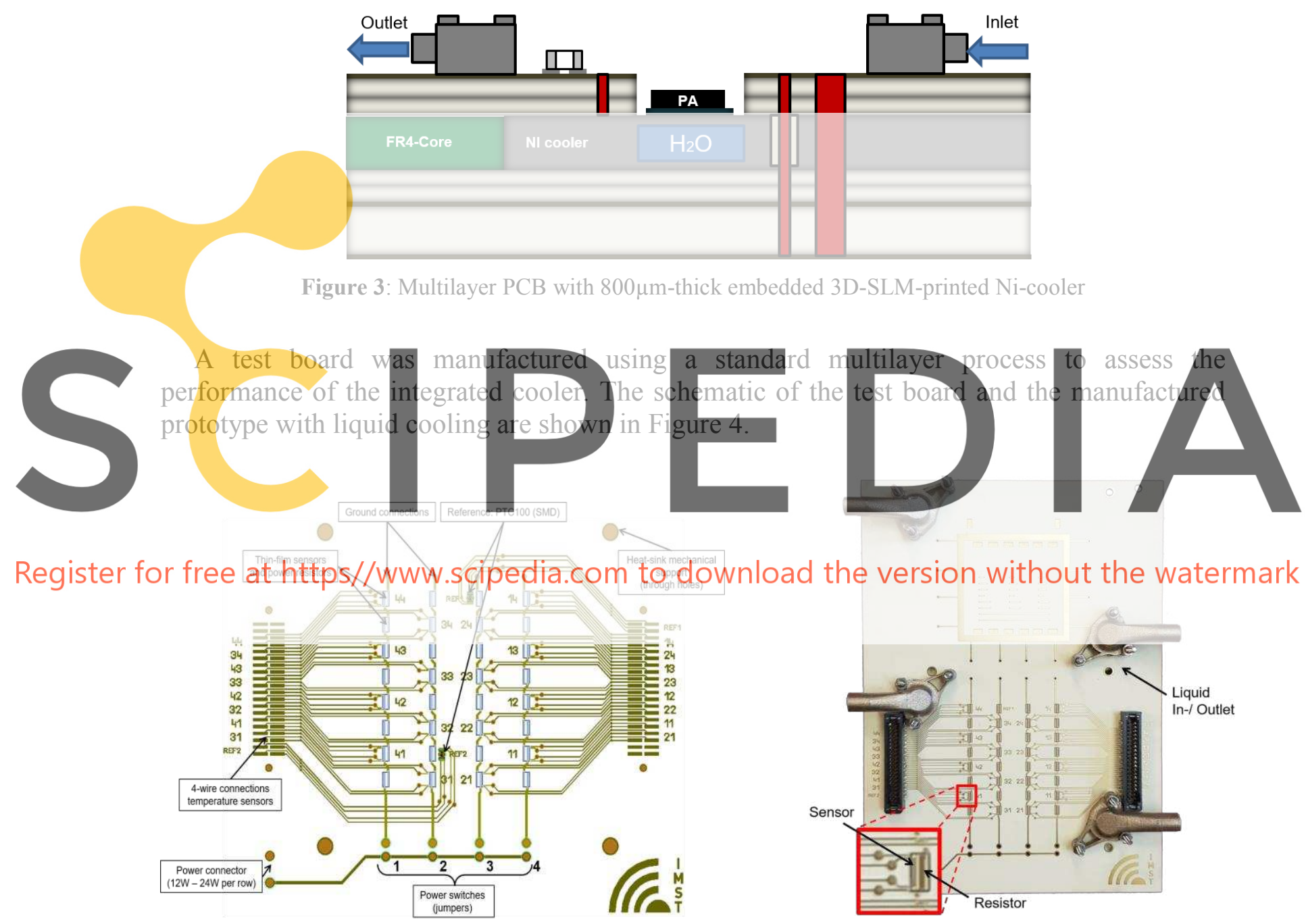

Figure 4: Left: Layout schematic of the thermal test boards; Right: Thermal test board with integrated liquid cooling

The inner core, in which the integrated Ni-cooler is embedded, is made out of standard FR4 material. Layers of Panasonic Megtron 6 RF-substrate (core and prepreg) are laminated onto it, to provide space for $\mathrm{RF}$ routing. $\mathrm{SiO} 2$ thin film resistors were glued to the surface of 
the cooler in cavities foreseen in the PCB. These resistors simulate the effect of the active components, especially the power amplifiers, on a real PCB. The thin film resistors were dimensioned to provide the same heat as the PAs to be used in the ACASIAS project. In total, 64 of these heat sources were assembled, distributed in four rows of 8 resistors. The dissipated power can be controlled by adjusting the current and voltage delivered to the circuit. Small temperature-dependent resistors were assembled in the vicinity of some of the thin-film resistors, to sense the temperature variations. Two PTC100 temperature sensors are clamped onto the PCB at the end of the Ni-cooler as a reference.

\section{MEASUREMENT RESULTS AND COMPARISON}

The performance of the thermal demonstrator was measured in a temperature-controlled environment. The obtained results were compared to those obtained for a similar test board with a conventional heat dissipation strategy, namely a copper core embedded in the PCB stack. As a first step, a single row of resistors was activated. The temperature on the PCB was monitored using a thermal imaging camera and the temperature sensors assembled in the PCB. Figure 5 shows the heat distribution on the PCB, after switching on the resistors behaviour (left) and after switching on the pump for the liquid cooling. It is clearly seen that the liquid cooling of the PCB has a very quick temperature response and a high cooling capability. The thermal images show that the cooling system is both quick and effective.
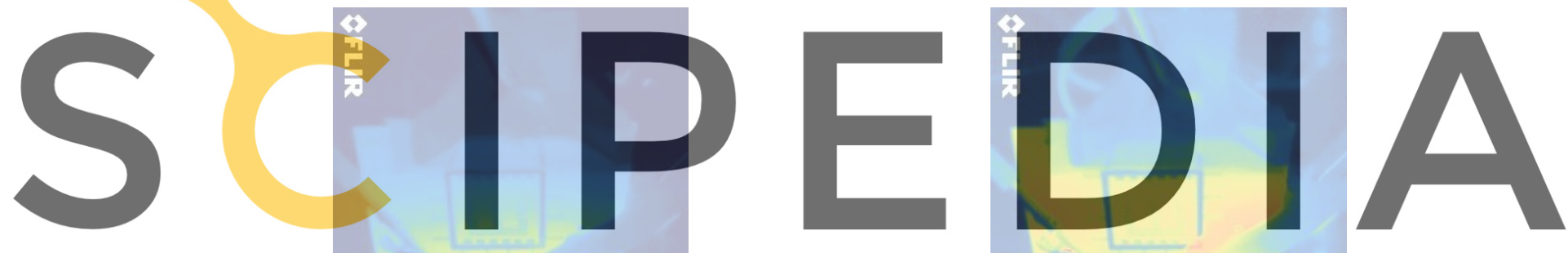

Register for free at https//www.scipedia.com to download the

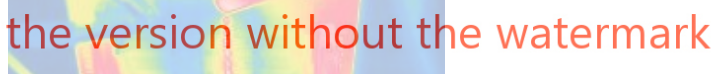

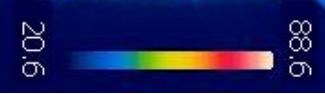

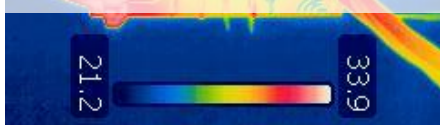

Figure 5: Temperature behaviour of the demonstrator after turning on one row of resistors, before (left) and 5 seconds after turning on the liquid cooling system (right). $12 \mathrm{~W}$ dissipated power, 1 bar pressure (cooling fluid.)

Figure 6 (left) displays the evolution of the temperature detected by the on-board sensors as a function of time. The sensors T11- T14 are located along the active row, while T22 - T24 are along the parallel row, about $1 \mathrm{~cm}$ away from the active heating components. The results show how the temperature quickly raises from the moment the resistors are activated on until $t=220 \mathrm{~s}$, when the cooling system is turned on. At that point, the maximum temperature in the PCB has reached the steady state of $90^{\circ} \mathrm{C}$. The temperature decreases quickly once the liquid cooling is activated, to reach a steady state of $38^{\circ} \mathrm{C}$. Increasing the thermal dissipation does not significantly increase this steady state temperature, as shown in Figure 6 (right): multiplying the power by 4 , to $48 \mathrm{~W}$, causes only $1{ }^{\circ} \mathrm{C}$ increase in temperature; doubling it 
again to $96 \mathrm{~W}$ shifts the steady state to only $51^{\circ} \mathrm{C}$, which is still within the operating margin of MMIC components.
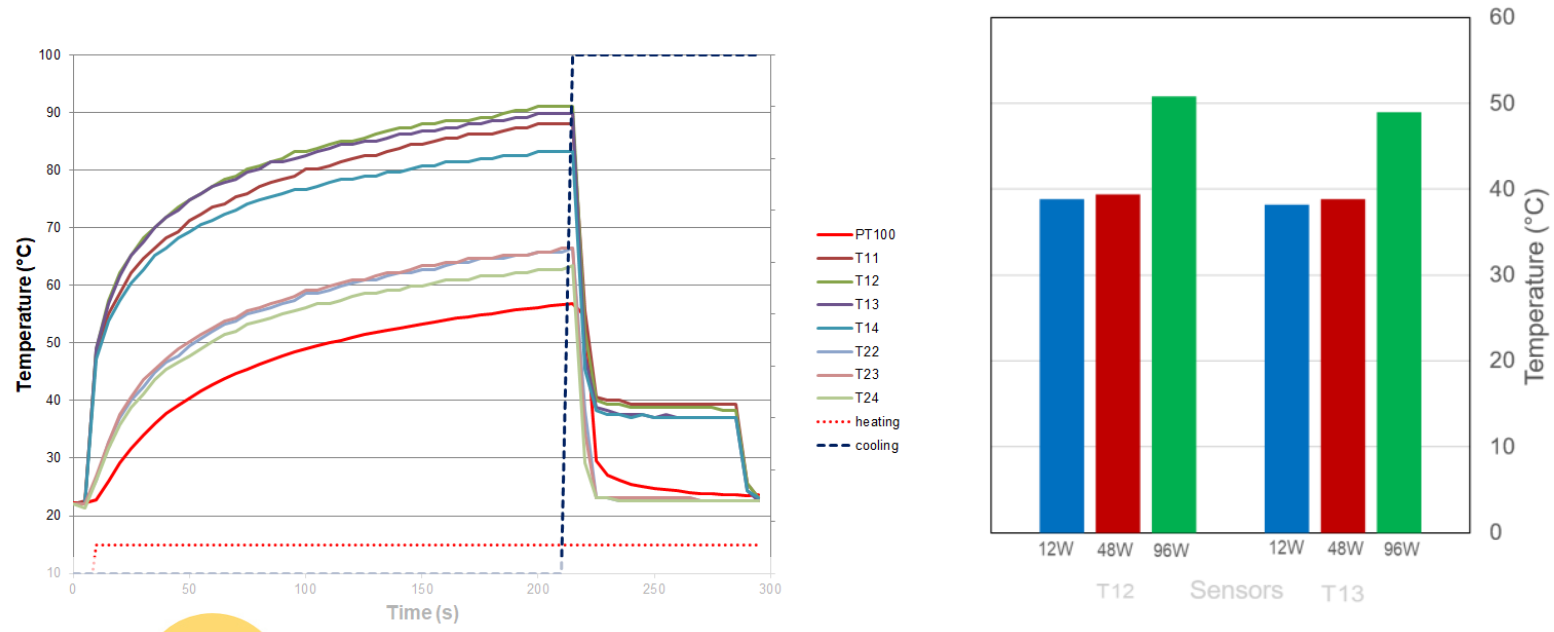

Figure 6: Left: Temperature detected by the on-board temperature sensors, with one row activated, $12 \mathrm{~W}$ dissipated power; Right: Temperature detected by the sensors T12 and T13 vs. dissipated power.

A comparison, the experiment was repeated with the same layout, but on a PCB with a copper core. In this second case, the maximum temperature reached without additional cooling is around $73^{\circ} \mathrm{C}$, clearly lower than the $90^{\circ} \mathrm{C}$ reached with the previous PCB. This is due to the fact that the copper PCB core has a high thermal conductivity, and spreads the heat over the whole area of the PCB. Figure 7 and Figure 8 shows the performance comparison between the two thermal demonstrators: copper core, a rib-cooler and a fan and with the liquid cooling for the same level of applied power ( $48 \mathrm{~W}$, four active rows), and the same ambient conditions. The liquid cooling allows decreasing the PCB temperature $34^{\circ} \mathrm{C}$ more than the passive conventional solution. The copper core, in this case, has reached the limit of

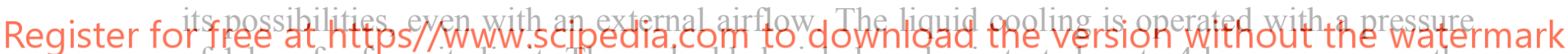
of 1 bar, far from its limit: The embedded nickel cooler is tested up to 4 bar pressure, so the device has much cooling reserve left.
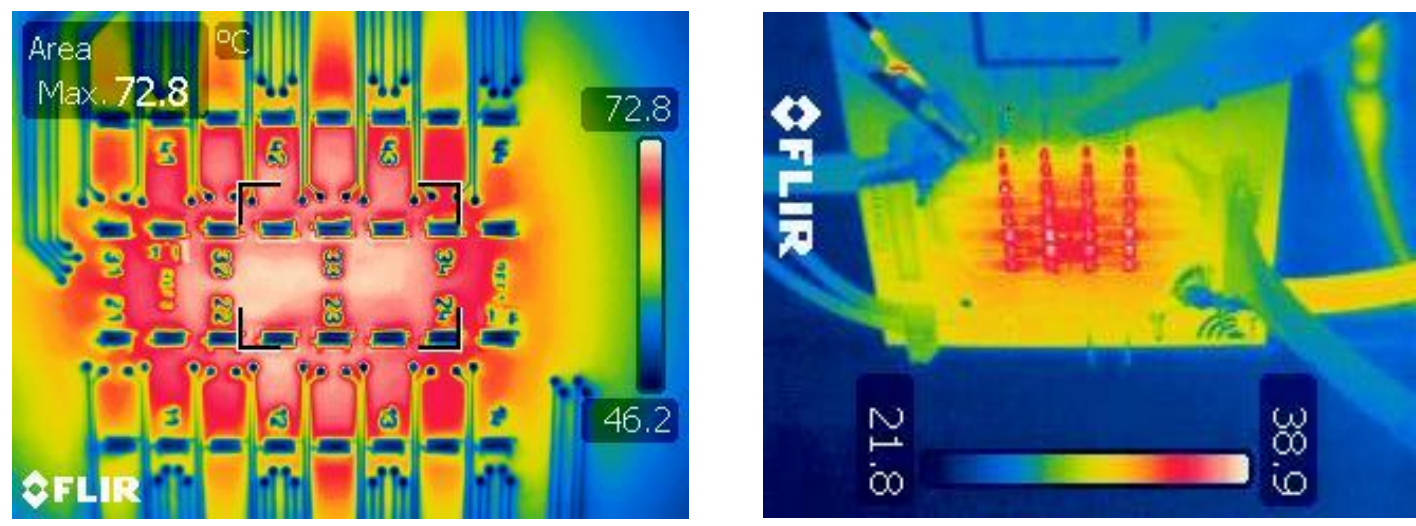

Figure 7: comparison between conventional copper core PCB with rib-cooler \& fan (left) and PCB with liquid cooling system (right), 4 active rows, $48 \mathrm{~W}$ dissipated power, 1 bar pressure (cooling fluid.) 


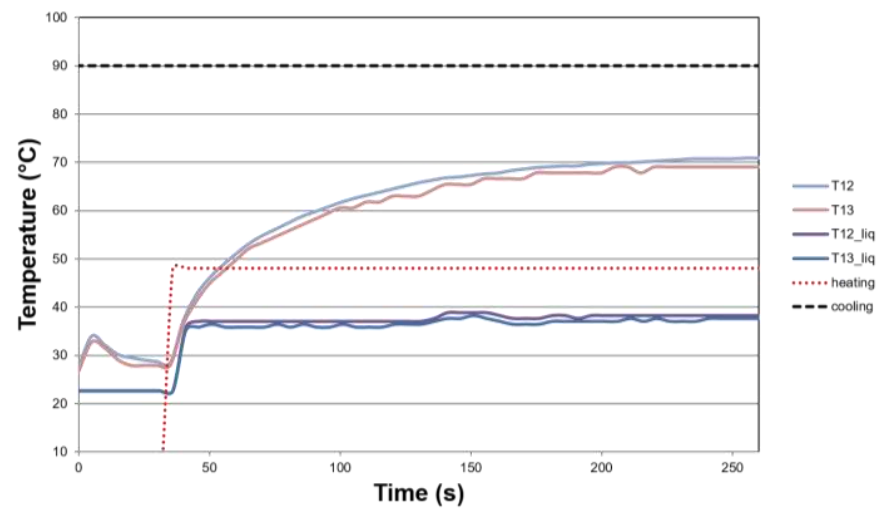

Figure 8: Performance comparison between the PCB with copper core vs. PCB with liquid cooling

The results highlight the superior cooling performance of the integrated Ni-cooler for the heat management of the PCB. It allows reaching quickly the steady state, so that the working point of the RF-circuits can be adjusted.

\section{CONCLUSIONS}

In this paper the manufacturing and measurements off an innovative embedded PCBliquid cooling is presented. It shows the high potential of this technology for future buildups of active phased array antennas with a focus of on power dissipation and RF-routing capabilities. It is shown that such a device is manufacturable in a standard multilayer PCB technology. The codlin and applications, such

ACKNOWLED GEMENT

The work described in this paper has received funding from the European Union's Horizon 2020 research and innovation programme under grant agreement. No 723167. ACASIAS

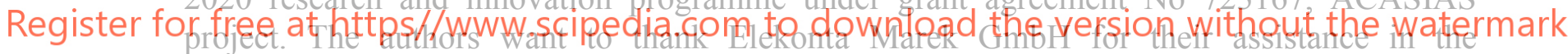
manufacturing of the prototypes, and Uwe Gollor and Winfried Simon for their help with the thermai measurements.

\section{REFERENCES}

[1] "Advanced Concepts for Aero-Structures with Integrated Antennas and Sensors", http://www.acasias-project.eu/

[2] Ebert, T., „Metallkühler für Leistungsbauteile aus dem 3D-Laserdrucker“, Elektronik Praxis: Sonderheft Leistungselektronik (2018), 20 -22.

[3] Wienhausen, A.H., Sewergin, A., de Doncker, R., Highly Integrated Two-Phase SiC Boost Converter with 3D Printed Fluid Coolers and 3D Printed Inductor Bobbins, Proc. PCIM Europe; Int. Exhibition \& Conf. Power Electr., Intelligent Motion, Renewable Energy \& Energy Mgmt (2018) 1-8.

[4] Curran, B., Reyes, J., Tschoban, C., Höfer, J., Grams, A., Wüst, F., Hutter, M., Leiß, J., Martinez-Vazquez, M., Baggen, R., Ndip, I., Lang, K-D., "Development and Validation of a Chip Integration Concept for Multi-Die GaAs Front-Ends for Phased Arrays up to 60 GHz", IEEE Trans. Comp., Packaging \& Manufacturing Techn. (2018), 8:1231-1240. 